\title{
Management of Adverse Reactions to Iodinated Contrast Media for Computed Tomography in Korean Referral Hospitals: A Survey Investigation
}

\author{
Seungchul Han, $M D^{1}$, Soon Ho Yoon, $M D^{1,2}$, Whal Lee, MD, $P D^{1,2}$, Young-Hun Choi, $M D^{1,2}$, \\ Dong Yoon Kang, $\mathbf{M D}^{3,4,5}$, Hye-Ryun Kang, $\mathbf{M D}^{3,4,5}$ \\ Departments of ${ }^{1}$ Radiology and ${ }^{5}$ Internal Medicine, Seoul National University College of Medicine, Seoul, Korea; ${ }^{2}$ Institute of Radiation Medicine, \\ Seoul National University Medical Research Center, Seoul, Korea; ${ }^{3}$ Drug Safety Monitoring Center, Seoul National University Hospital, Seoul, Korea; \\ ${ }^{4}$ Seoul National University Hospital Regional Pharmacovigilance Center, Seoul, Korea
}

Objective: To evaluate the current status of managing adverse reactions to iodinated contrast media (ICM) for computed tomography in referral hospitals in South Korea compared with hospitals in other countries.

Materials and Methods: This survey investigation involved 59 Korean and 15 overseas hospitals using guideline-based questionnaires consisting of 24 items in 7 main categories related to managing adverse reactions to ICM.

Results: Informed written consent with risk factor evaluation was appropriately performed in most of the Korean hospitals. There was considerable variability in assessing renal function across the hospitals; serum creatinine level was used as a reference in $76.4 \%$ of Korean hospitals. The Korean hospitals preferred a more stringent approach to determining normal renal function $(p=0.01)$, withholding metformin $(p=0.01)$, and fasting before ICM exposure $(p<0.001)$ compared with overseas hospitals. All the Korean hospitals had an emergency protocol and in-hospital system for adverse reactions to ICM. The Korean $(87.7 \%)$ and overseas hospitals $(100 \%)$ were similarly equipped with epinephrine $(p=0.332)$, but only $38.6 \%$ of Korean hospitals were equipped with a bronchodilator $(p=0.004)$. For patients with a previous hypersensitivity reaction to ICM, $62.3 \%$ of Korean hospitals pre-medicated with anti-histamine and corticosteroid according to the severity of the previous reaction, and changed the culprit ICM in $52.8 \%$, while skin test was performed in $17 \%$.

Conclusion: In general, Korean referral hospitals were well-prepared regarding informed consent, protocol, and an inhospital system for managing adverse reactions to ICM. Nevertheless, there was considerable variability in details and management, thus requiring standardization by reflecting current guidelines.

Keywords: Guideline; Radiology practice; Emergency protocol; Drug allergy; Asthma

\section{INTRODUCTION}

Iodinated contrast media (ICM) is an essential pharmaceutical in contemporary radiology, with approximately 75 million administrations per year worldwide (1). The use of ICM is generally regarded to be safe. However, acute adverse reactions to current non-ionic ICM occur in $0.7-3.1 \%$ of patients and, although most reactions are confined to mild urticaria, they can be life-threatening $(2,3)$. To manage adverse reactions to ICM, several clinical guidelines have been published based on recent studies and consensus statements by relevant expert committees such as the American College of Radiology (ACR), European Society of Urogenital Radiology (ESUR) $(4,5)$, and Korean Network of Allergists \& Radiologists for Research on Radiocontrast media-associated Adverse reactions (6). The

Received December 21, 2017; accepted after revision May 21, 2018.

This study was supported by a grant (17172MFDS158) from Korea Ministry of Food and Drug Safety in 2017.

Corresponding author: Soon Ho Yoon, MD, Department of Radiology, Seoul National University College of Medicine, 101 Daehak-ro, Jongno-gu, Seoul 03080, Korea.

- Tel: (822) 2072-2057 • Fax: (822) 743-6385• E-mail: yshoka@gmail.com

This is an Open Access article distributed under the terms of the Creative Commons Attribution Non-Commercial License (https:// creativecommons.org/licenses/by-nc/4.0) which permits unrestricted non-commercial use, distribution, and reproduction in any medium, provided the original work is properly cited. 
guidelines were generally individualized to each hospital for managing the institutional radiology practice.

In Korea, the reported number of adverse reactions to ICM has been increasing annually. ICM occupied third place among the most common causative drugs for adverse drug reactions, with 18240 cases in 2016, following anticancer drugs and nonsteroidal anti-inflammatory drugs (7). Moreover, 25 cases of fatal reactions to ICM have been reported in South Korea in the past 5 years. The appropriate management of adverse reactions to ICM is growing in importance, especially in Korean referral hospitals, where several types of ICM are administered; however, to date, their current institutional management has not been assessed. Accordingly, the aim of the present study was to evaluate the current status of managing adverse reactions to ICM in Korean referral hospitals compared with overseas hospitals.

\section{MATERIALS AND METHODS}

\section{Study Subjects}

This questionnaire-based investigation was conducted from May to September 2017 at 59 of 79 Korean tertiary or secondary referral training hospitals; 20 hospitals were excepted due to a lack of reply or inaccessibility. To compare trends in guidelines for managing adverse reactions to ICM in overseas hospitals, a convenience sample of 15 overseas hospitals, which were chosen through the authors' professional connections, were surveyed.

\section{Questionnaire}

The questionnaire (Supplementary Materials in the onlineonly Data Supplement) was developed by the authors, who are radiologists and allergists, based on current Korean and international guidelines for the use of $\operatorname{ICM}(5,6,8)$. It had a total of 24 items and consisted of 7 main categories: baseline institutional characteristics; informed consent and risk factor identification before ICM injection; pretest screening of renal function; policy for withholding metformin; preparative fasting for ICM injection; system and protocol for managing adverse reactions to ICM; and management of patients with a history of a previous allergic reaction to ICM. Some were multiple-choice questions, and some were answered "yes" or "no." In the multiple-choice items, the responder could freely compose an answer in a blank at the end of the list if an appropriate answer was not offered.

\section{Statistical Analysis}

For statistical analysis, the answers were anonymized, and were not used for any other purposes. Binary or multiple-choice items were summarized in proportion. Answers representing continuous variables are expressed as median with interquartile range (IQR). Korean and overseas hospitals were analyzed separately and compared with one another. Fisher's exact test, Pearson's chi-squared test, and the Mann-Whitney U test were used to compare responses. All analyses were performed using SPSS version 22.0 (IBM Corp., Armonk, NY, USA).

\section{RESULTS}

\section{Baseline Institutional Characteristics}

Among the 59 Korean hospitals surveyed, 55.9\% (33/59) are located in the city of Seoul or Gyeonggi province, $28.8 \%(17 / 59)$ are in other metropolitan cities, and the remaining $15.3 \%(9 / 59)$ are in other provinces (Fig. 1). Tertiary referral institutions comprised $62.7 \%$ (37/59) of the hospitals, while $37.3 \%(22 / 59)$ were secondary referral hospitals. Among the 15 overseas hospitals surveyed, $73.3 \%(11 / 15)$ were located in Asia (Japan [ $n=2]$; China $[n=2]$; Hong Kong $[n=1]$; Jordan $[n=1] ;$ Myanmar [ $n$ $=2]$; Taiwan $[n=2]$; Vietnam $[n=1]), 13.3 \%(2 / 15)$ in America (Canada $[n=1]$; Cayman Islands $[n=1]$ ), and the remaining $13.3 \%(2 / 15)$ were in Europe (France $[n=2])$.

The median number of computed tomography (CT) scanners was 3 (IQR 2-4) in the Korean hospitals and 4.5 (IQR 3-5.5) in the overseas hospitals ( $p=0.305$ ). Most of the Korean and overseas hospitals performed at least 50-100 CT scans per day (Fig. 2). Of the Korean hospitals, $45.8 \%$ had $\geq 5$ types of low- or iso-osmolar ICM, as determined by their generic names, in contrast to the overseas hospitals, among which $40.0 \%$ had 2 types of ICM available $(p=0.001)$ (Fig. 3$)$. There was a significant difference between the tertiary and secondary referral hospitals in the number of CT scanners (median 4 vs. 2.5; $p<0.001)$, the number of CT scans performed per day ( $p=$ $0.002)$ and the number of types of contrast agents available $(p=0.019)$.

Informed Consent and Risk Factor Identification before ICM Injection

All $(100 \%)$ Korean hospitals and $93.3 \%$ of overseas hospitals had a standard consent form, and also provided them to their patients to obtain written informed consent 


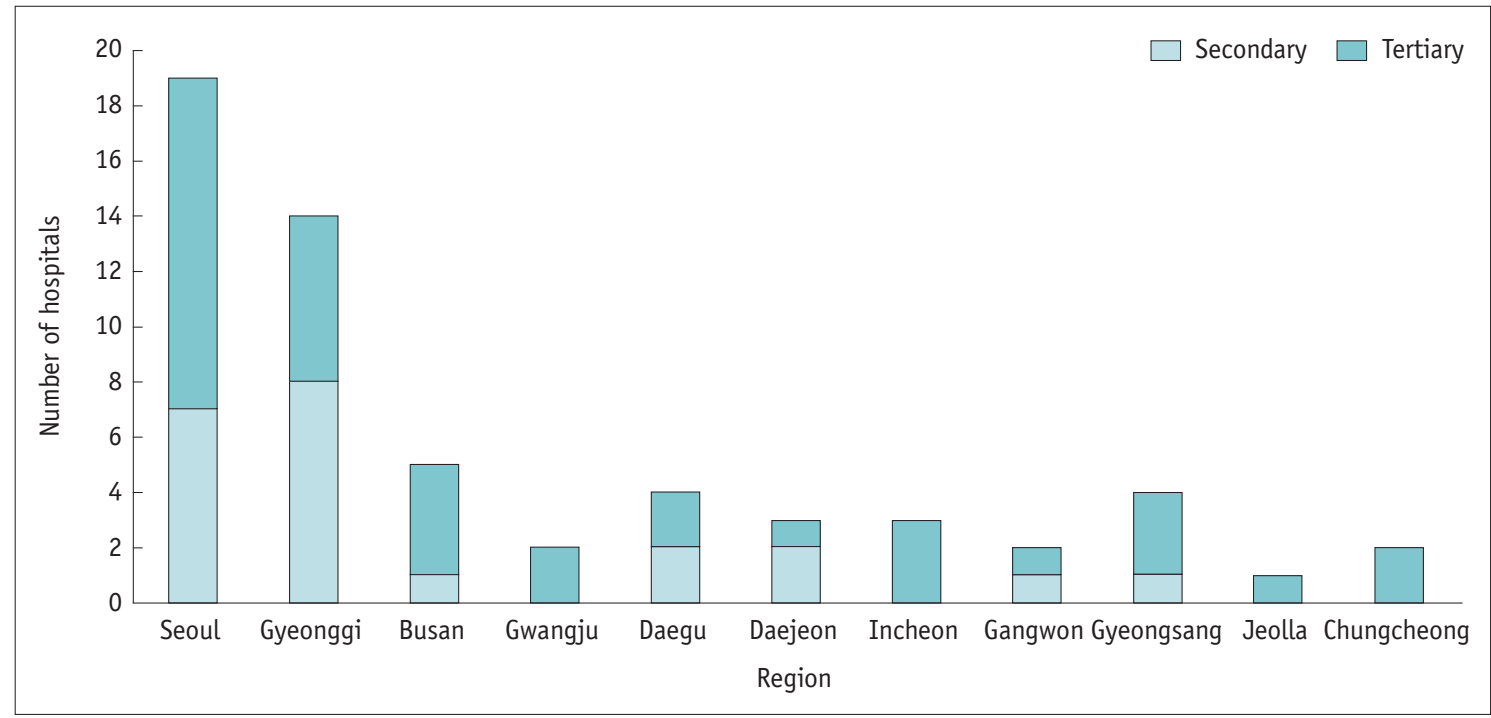

Fig. 1. Distribution of tertiary and secondary referral hospitals in Korea.

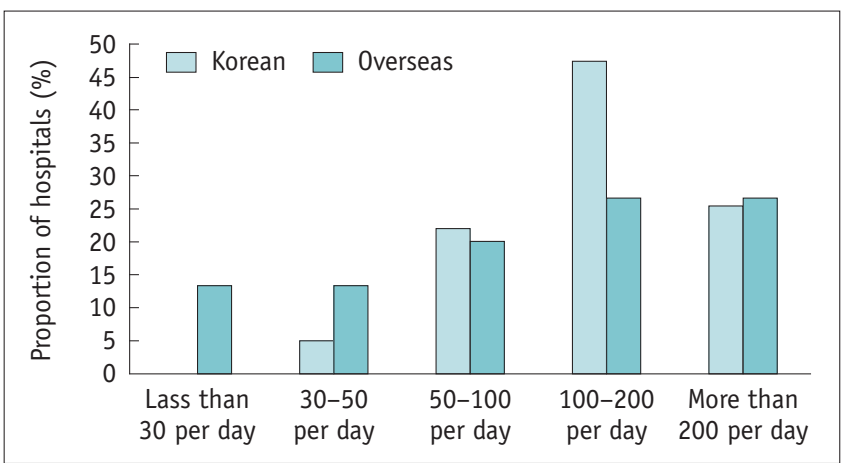

Fig. 2. CT scans performed per day in hospitals. CT = computed tomography

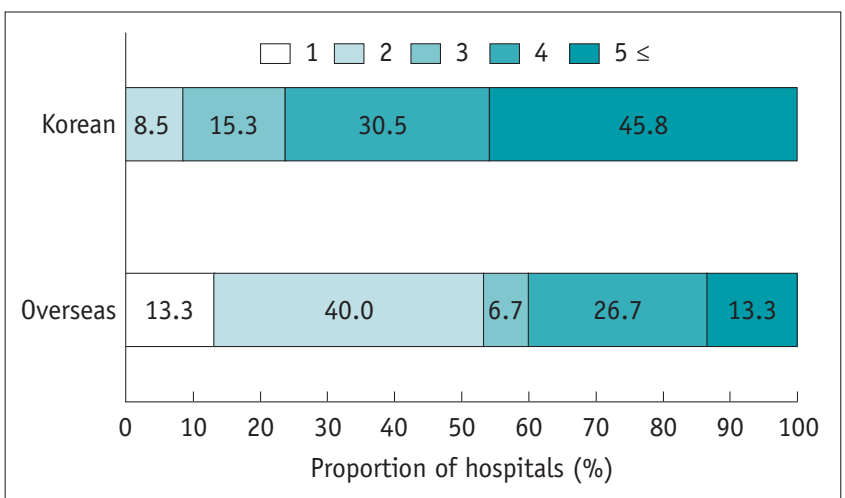

Fig. 3. Distribution of number of ICM types used in hospitals. ICM = iodinated contrast media

before undergoing ICM-enhanced CT scans (Korean hospitals, $100 \%$; overseas hospitals, $86.7 \%$ ).

All the Korean hospitals evaluated patient risk factors before ICM injection. The most commonly surveyed factors were the presence of renal disease $(100 \%)$ and a history of a hypersensitivity reaction after contrast-enhanced CT scan $(98.3 \%)$, followed by drug allergy $(94.8 \%)$, diabetes mellitus $(94.8 \%)$, history of previous exposure to ICM $(89.7 \%)$, asthma (84.5\%), hypertension $(79.3 \%)$, cardiac diseases $(74.1 \%)$, and hyperthyroidism (50.0\%) (Table 1$)$. Similar trends were observed in the overseas hospitals, except for questions addressing hypertension and cardiac disease. Some of the overseas hospitals also took into account other factors, such as myasthenia gravis and pheochromocytoma.

\section{Pre-Test Screening of Renal Function}

Patient renal function was evaluated based on laboratory findings in $93.2 \%$ (55/59) of the Korean hospitals: $63.6 \%$ $(35 / 55)$ of these hospitals used one screening test and $36.4 \%(20 / 55)$ required 2 laboratory tests. The serum creatinine level was used in $76.4 \%(42 / 55)$ of the Korean hospitals, while estimated glomerular filtration rate (eGFR) was used in $50.9 \%(28 / 55)$. The cut-off for serum creatinine level was heterogeneously distributed across the Korean hospitals, with a median value of $1.2 \mathrm{mg} / \mathrm{dL}(1.4 \mathrm{mg} / \mathrm{dL}$ in overseas hospitals; $p=0.53$ ) (Fig. $4 \mathrm{~A}$ ). The median cut-off for eGFR was $60 \mathrm{~mL} / \mathrm{min} / 1.73 \mathrm{~m}^{2}$ in Korean hospitals, which was significantly higher than that in overseas hospitals (30 $\mathrm{mL} / \mathrm{min} / 1.73 \mathrm{~m}^{2}(p=0.01)$ (Fig. 4B).

\section{Policy on Withholding Metformin}

Nearly all hospitals, 98.2\% (55/56) in Korea and 93.3\% $(14 / 15)$ overseas, routinely verified whether the patient was taking metformin before ICM injection. Among the 
hospitals, $57.1 \%(32 / 56)$ of those in Korea and $53.3 \%$

(8/15) of those outside Korea discontinued metformin, regardless of renal function. Serum creatinine or eGFR levels were used to consider discontinuing metformin in $48.2 \%$ (27/56) of Korean and 33.3\% (5/15) of overseas hospitals. Among Korean hospitals, a significantly larger proportion of secondary referral hospitals (45.5\% [10/22]) used eGFR level as a reference than tertiary hospitals $(17.6 \%[6 / 34])$ $(p=0.024)$. Only $5.4 \%(3 / 56)$ of Korean and $13.3 \%(2 / 15)$ of overseas hospitals continued metformin irrespective of ICM injection. The cut-off for serum creatinine was, again, heterogeneously distributed across the Korean hospitals,

Table 1. Risk Factors for Evaluation before Undergoing Contrast-Enhanced CT Scan

\begin{tabular}{|c|c|c|c|}
\hline & Korean $(n=58)(\%)$ & Overseas $(n=15)(\%)$ & $P$ \\
\hline History of previous exposure of ICM & $52(89.7)$ & $12(80.0)$ & 0.379 \\
\hline $\begin{array}{l}\text { History of hypersensitivity reaction after } \\
\text { contrast-enhanced CT scan }\end{array}$ & $57(98.3)$ & $15(100.0)$ & 1.000 \\
\hline Drug allergy & $55(94.8)$ & $15(100.0)$ & 1.000 \\
\hline Asthma & $49(84.5)$ & $13(86.7)$ & 1.000 \\
\hline Diabetes mellitus & $55(94.8)$ & $14(93.3)$ & 1.000 \\
\hline Hypertension & $46(79.3)$ & $5(33.3)$ & $0.001^{*}$ \\
\hline Renal disease & $58(100.0)$ & $14(93.3)$ & 0.205 \\
\hline Cardiac disease & $43(74.1)$ & $6(40.0)$ & $0.028 *$ \\
\hline Hyperthyroidism & $29(50.0)$ & $9(60.0)$ & 0.570 \\
\hline Others & $8(13.8)$ & $6(40.0)$ & $0.032 *$ \\
\hline
\end{tabular}

*Statistical significance. Others included pregnancy, smoking, operation history, airway disease, and bleeding tendency. CT = computed tomography, ICM = iodinated contrast media

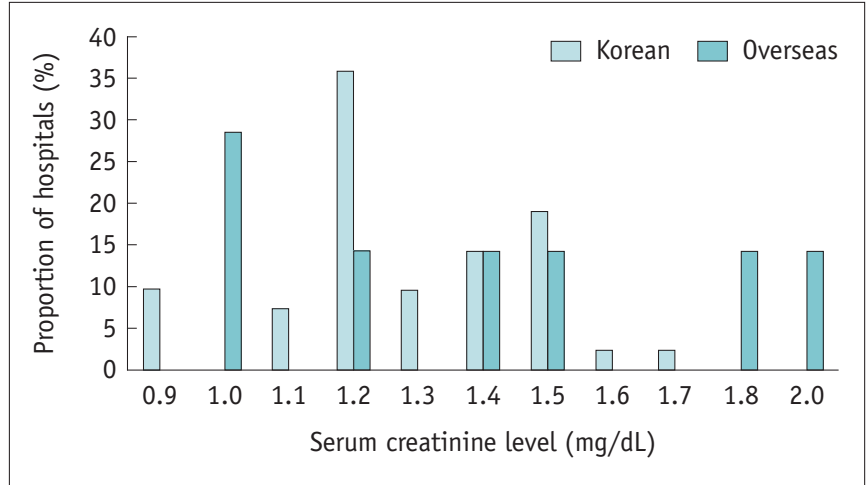

A

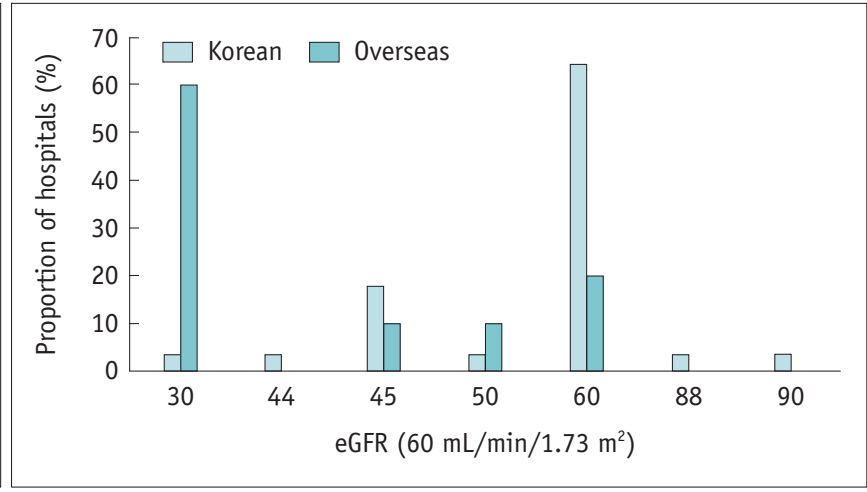

B

Fig. 4. Cut-off level for serum creatinine (A) and eGFR (B) for contrast-enhanced CT scans in Korean hospitals. eGFR = estimated glomerular filtration rate

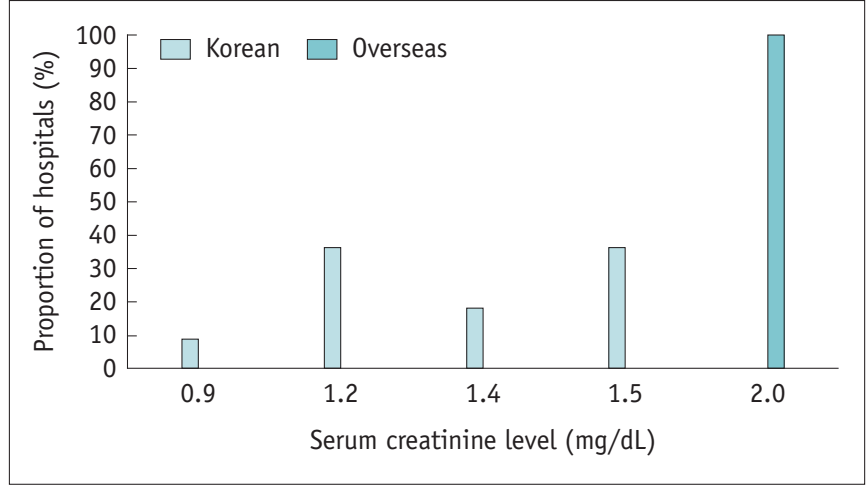

A

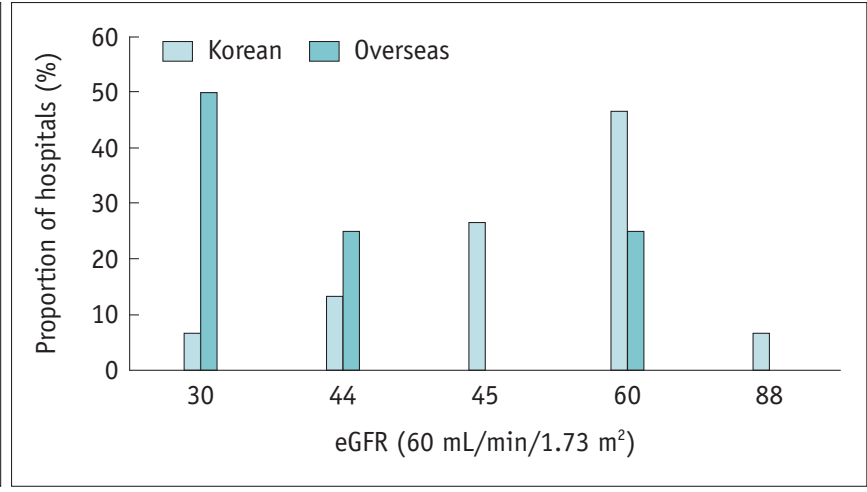

B

Fig. 5. Cut-off level for serum creatinine (A) and eGFR (B) for stopping metformin before contrast-enhanced CT scans in Korean hospitals. 


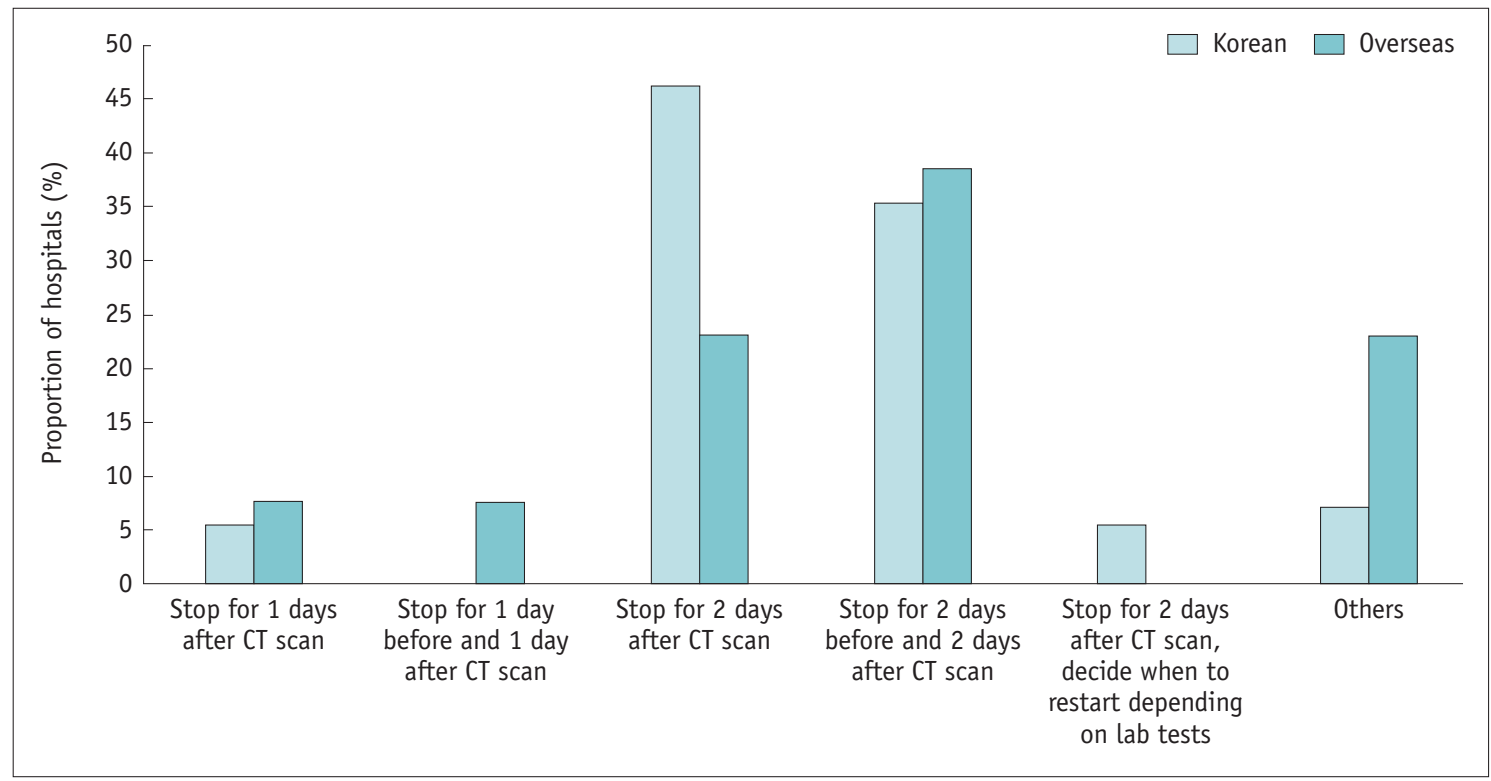

Fig. 6. Distribution of duration of withholding metformin. Other answers included "Restart by physician," "Restart at any time after CT scan depending on lab results," etc.

with a median value of $1.4 \mathrm{mg} / \mathrm{dL}$ (Fig. 5A), whereas only 1 overseas hospital used serum creatinine as the reference, with a cut-off of $2 \mathrm{mg} / \mathrm{dL}(p=0.17)$. The median cut-off level for eGFR was $60 \mathrm{~mL} / \mathrm{min} / 1.73 \mathrm{~m}^{2}$ in Korean hospitals, which was significantly higher than that in overseas hospitals ( $44 \mathrm{~mL} / \mathrm{min} / 1.73 \mathrm{~m}^{2}(p=0.01)$ (Fig. 5B).

Among the hospitals requiring withholding of metformin, hospitals accounting for the largest proportion (46.3\% [25/54]) of Korean hospitals discontinued for 2 days after the CT scan, and hospitals accounting for the largest proportion $(38.5 \%[5 / 13])$ of overseas hospitals was discontinued 2 days before the CT scan until 2 days after (Fig. 6).

\section{Preparative Fasting before ICM Injection}

Among the Korean hospitals, 98.3\% (57/58) required patients to fast before ICM injection compared with $80.0 \%$ $(12 / 15)$ of the overseas hospitals $(p=0.005)$. Among the hospitals that required fasting, $58.9 \%(33 / 56)$ of the Korean hospitals required fasting from both solid food and water, in contrast to the overseas hospitals, in which $83.3 \%$ $(10 / 12)$ required fasting from only solid food $(p=0.007)$ (Fig. 7). The median fasting time required in Korean and overseas hospitals was 6 hours and 4 hours, respectively, for both solid food $(p<0.001)$ and water $(p=0.003)$.

\section{System and Protocol for Managing Adverse Reactions} to ICM

In case of an adverse reaction to ICM, 98.3\% (57/58) of

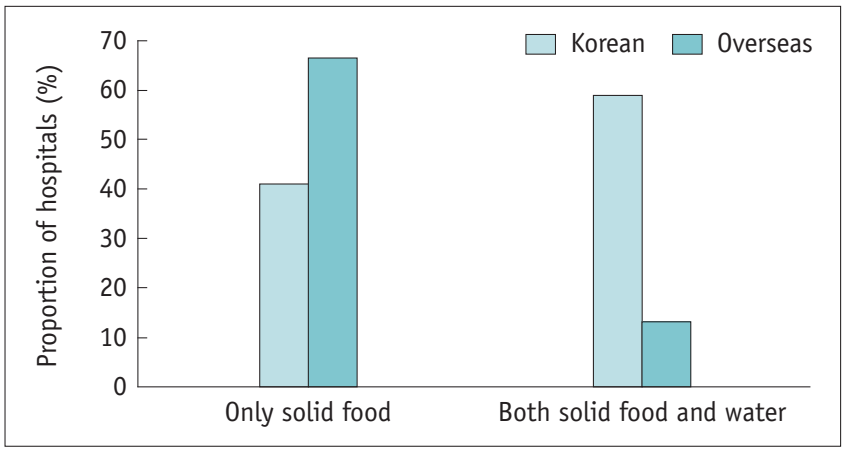

Fig. 7. Contents of pre-scan fasting.

the Korean hospitals and $93.3 \%(14 / 15)$ of the overseas hospitals had emergency agents in the CT room, including epinephrine, albuterol sulfate inhalation aerosol (Ventolin), chlorpheniramine and steroids, and equipment such as oxygen masks, an intubation kit and defibrillators, and other drugs such as atropine and lidocaine. Significantly fewer Korean hospitals had Ventolin (38.6\% [22/57]) in their CT room than did the overseas hospitals $(80.0 \%$ [12/15]) $(p=0.004)$ (Table. 2). All (100\% [15/15]) of the overseas hospitals were equipped with epinephrine compared with $87.7 \%(50 / 57)$ of Korean hospitals; this difference, however, was not statistically significant $(p=$ 0.332). During night-shift hours, $36.2 \%(21 / 58)$ of Korean hospitals did not have accompanying medical staff when administering ICM, but only $6.7 \%(1 / 15)$ of the overseas hospitals did not $(p=0.026)$. At the overseas hospitals, most of the accompanying medical staff were radiologists 
Table 2. Emergency Equipment in CT Room

\begin{tabular}{lccc}
\hline & Korean $(\mathrm{n}=57)(\%)$ & Overseas $(\mathrm{n}=15)(\%)$ & $P$ \\
\hline Epinephrine & $50(87.7)$ & $15(100)$ & $12(80.0)$ \\
Ventolin & $22(38.6)$ & $11(73.3)$ & 0.332 \\
Chlorpheniramine & $49(86.0)$ & $15(100.0)$ & $0.004^{*}$ \\
Steroids & $45(78.9)$ & $14(93.3)$ & 0.060 \\
Oxygen mask & $55(96.5)$ & $12(80.0)$ & 0.509 \\
Intubation kit & $50(87.7)$ & $11(73.3)$ & 0.425 \\
Defibrillator & $42(73.7)$ & $4(26.7)$ & 1.000 \\
Others (atropine, amiodarone, lidocaine) & $7(12.3)$ & 0.224 \\
\hline
\end{tabular}

*Statistical significance.

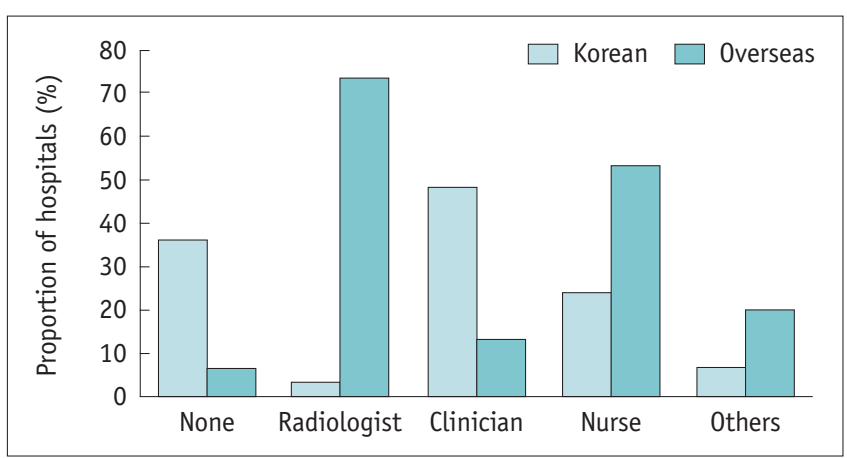

Fig. 8. Medical staff accompanying use of ICM for CT scanning during night-shift hours. Other answers included emergency department doctors, anesthetist, and intensive care unit doctors.

(73.3\%) and nurses (53.3\%), while in the Korean hospitals, most were clinicians in the corresponding department (48.3\%) (Fig. 8).

Adverse reactions to ICM were primarily managed by radiologists in $83.1 \%$ (49/59) of Korean and in $100 \%$ $(15 / 15)$ of overseas hospitals, followed by allergists in $37.3 \%(22 / 59)$ of Korean and $26.7 \%$ (4/15) of overseas hospitals. All (59/59) of the Korean hospitals and $86.7 \%$ $(13 / 15)$ of the overseas hospitals had an in-hospital system for recording and verifying adverse reactions to ICM. An electronic medical record (EMR) system was used by $89.8 \%$ $(53 / 59)$ of Korean and $84.6 \%$ (11/13) of overseas hospitals, and the remaining institutions used a separate system outside of the EMR or used paper charts. Hospitals without any in-hospital system relied on patient reports of adverse reactions at the time of written consent.

All the Korean and overseas hospitals had their own emergency protocols for managing adverse reactions to ICM. Their protocols varied, including different combinations of the following: severity categorization of the adverse reaction; emergency drug administration; resuscitation; guideline for management of delayed hypersensitivity; and guideline for the use of ICM in patients who experienced previous adverse reactions (Fig. 9). A significantly higher proportion of Korean hospitals (88.1\% [52/59]) than overseas hospitals $(46.7 \%$ [7/15]) had protocols for the further use of ICM $(p=0.001)$. Among Korean hospitals, only $78.4 \%$ (29/37) of tertiary hospitals had protocols for emergency drug administration compared with $100.0 \%$ $(22 / 22)$ in secondary hospitals $(p=0.02)$.

\section{Management of Patients with a History of a Previous Adverse Reaction to ICM}

Institutional protocols for patients with a history of an adverse reaction to ICM were in place in $91.5 \%$ (54/59) of the Korean hospitals and $73.3 \%(11 / 15)$ of the overseas hospitals $(p=0.076)$. The vast majority of the Korean and overseas hospitals included premedication with antihistamine and steroids (100\% and $90.9 \%$, respectively) and post-administration monitoring $(73.6 \%$ and $63.6 \%$, respectively) in their protocols. However, a skin test for selection of ICM was recommended in only small proportions of the Korean (17.0\% [9/53]) and overseas hospitals (18.2\% [2/11]) (Fig. 10). In fact, with regard to selection of ICM, a larger percentage of Korean hospitals (50.9\% [27/53]) replaced the previous causative agent with another without performing a skin test compared with the overseas hospitals $(18.2 \%$ [2/11]) $(p=0.036)$. Premedication was predominantly performed using antihistamine and corticosteroid according to severity of the previous adverse reaction in both Korean and overseas hospitals (62.3\% and $45.5 \%$, respectively). A smaller proportion of Korean and overseas hospitals performed premedication using antihistamine and steroid, regardless of the severity of the previous reaction $(17.0 \%$ and $18.2 \%$, respectively). Among the Korean hospitals, a significantly larger proportion of tertiary referral hospitals had medical staff accompany the CT scan compared with secondary hospitals $(26.5 \%$ and $0.0 \%$, respectively) $(p=0.019)$. With regard to 


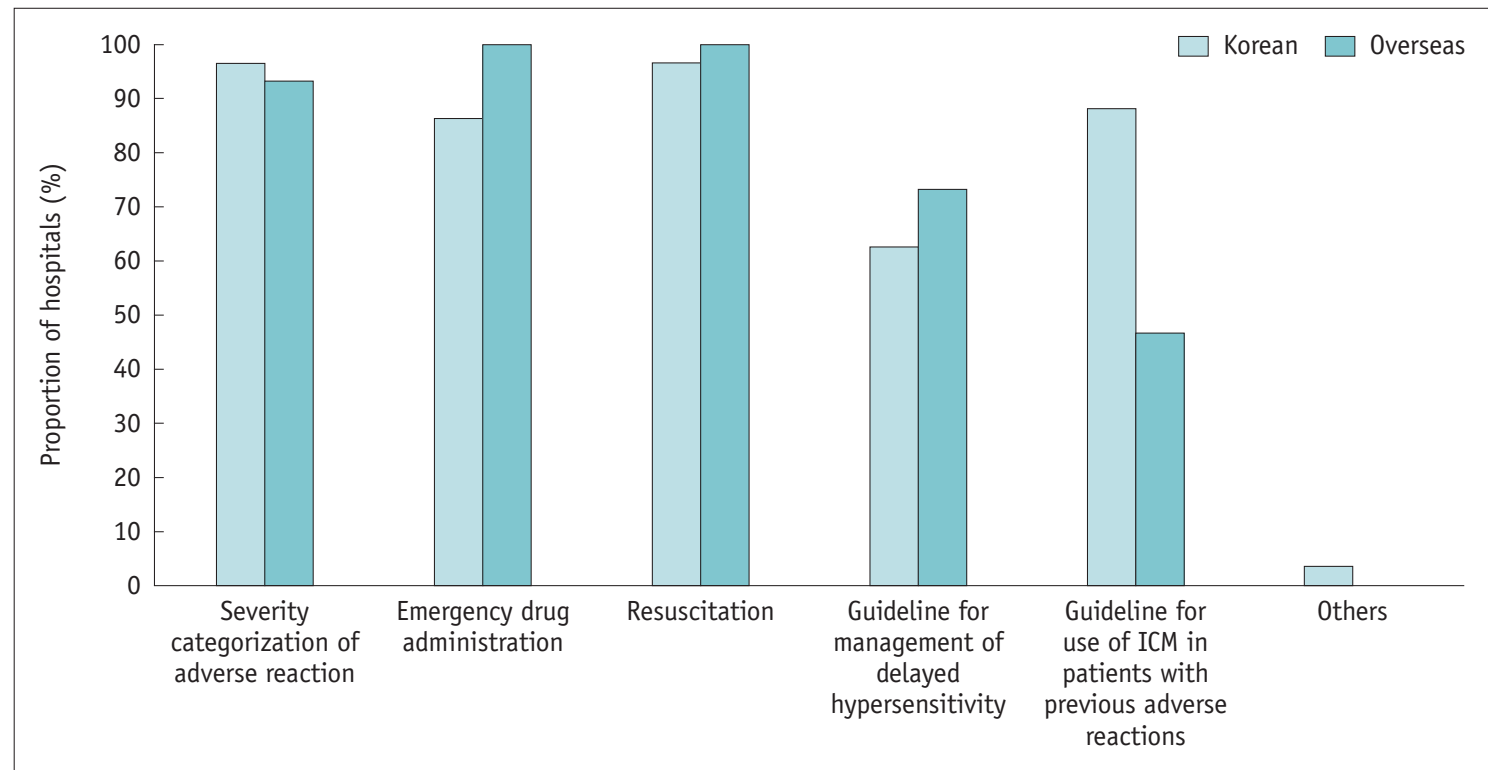

Fig. 9. Items included in emergency protocols for adverse reactions to ICM. Other answers included initial reporting system for adverse reactions.

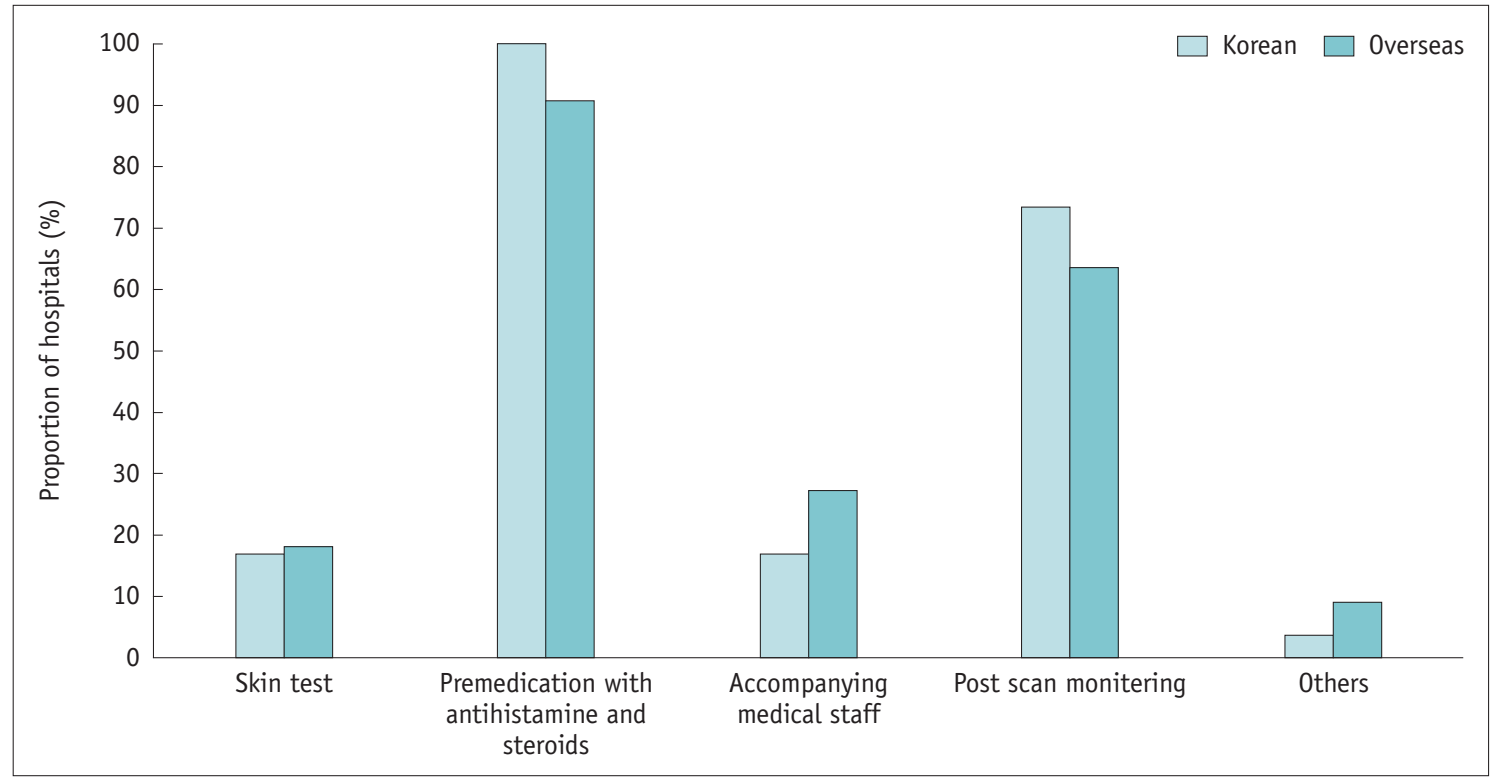

Fig. 10. Items included in pre-scan protocols for patients with previous adverse reactions to ICM. Other answers included reconsideration of proceeding $\mathrm{CT}$ scan.

premedication, $30.0 \%(6 / 20)$ of secondary referral hospitals administered antihistamine only, regardless of severity compared with $6.1 \%(2 / 33)$ of tertiary hospitals $(p=0.042)$. In the Korean hospitals without an institutional protocol (8.5\% [5/59]), 2 relied on the clinician's decision-making, 1 cancelled the examination, regardless of the severity of the previous event, and 2 either cancelled or required premedication depending on severity. In the overseas hospitals without a protocol (26.7\% [4/15]), 1 cancelled the examination, regardless of the severity of the previous event, and 3 cancelled the test when the previous event was severe (Fig. 11).

\section{DISCUSSION}

Our survey investigation was the first to examine the current status of managing adverse reactions to ICM in Korean referral hospitals, which is critical for the safety 


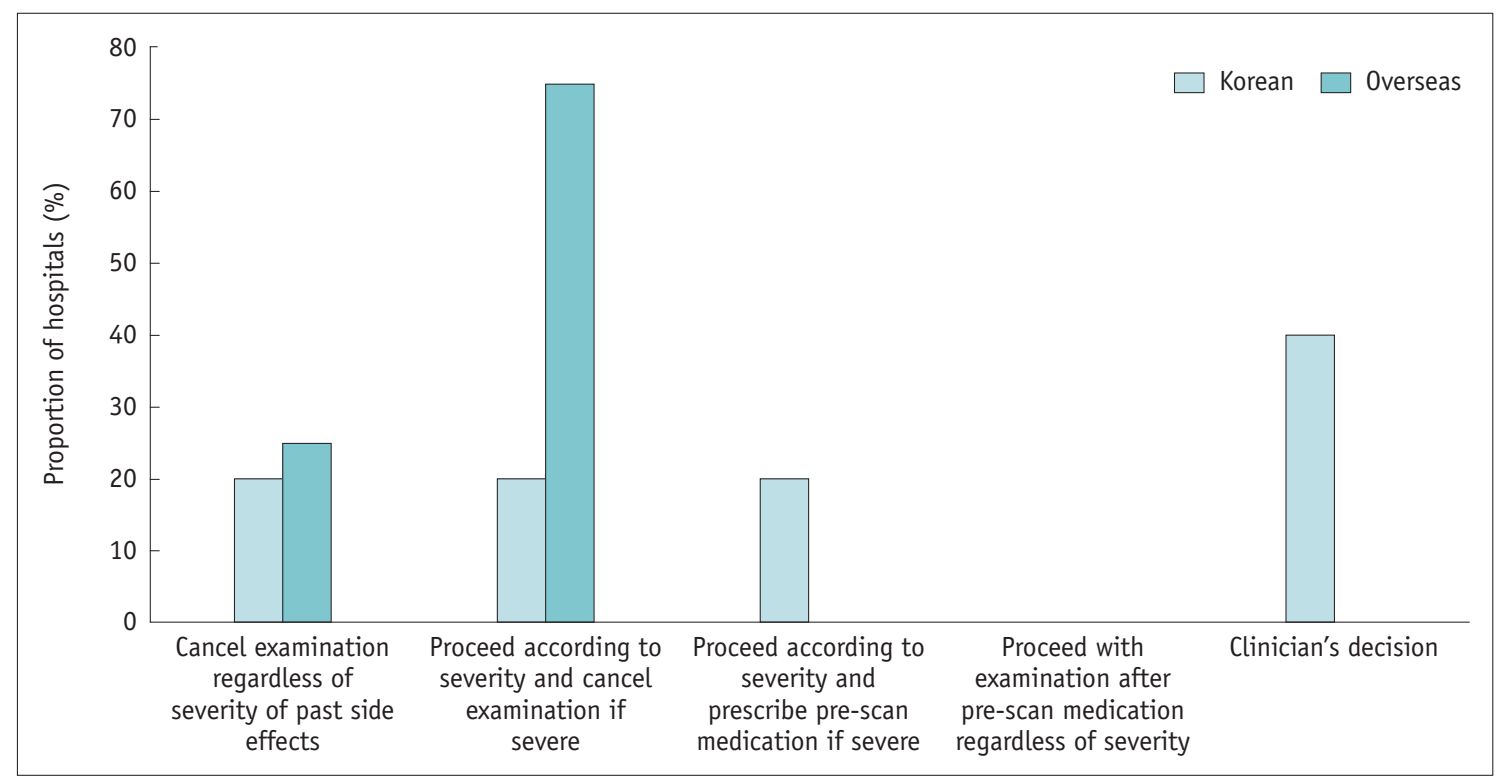

Fig. 11. Management of patients with previous adverse reactions to ICM in hospitals without protocols.

of patients who undergo ICM-enhanced CT. Most of the hospitals that participated in the survey prepared patients well before the administration of ICM in terms of written consent and risk factor evaluation (9). Their screened risk factors encompassed the majority of known risk factors for the 2 main types of adverse reactions to ICM: acute kidney injury and hypersensitivity reaction.

Although $93.2 \%$ of the Korean hospitals evaluated renal function based on a laboratory screening test, only $50.9 \%$ of hospitals adopted eGFR, which is a reference standard in the current guidelines. The majority of Korean hospitals verified serum creatinine level using varying cut-offs to identify normal renal function (6). In the Korean hospitals that had adopted eGFR, the median cut-off ( $60 \mathrm{~mL}$ ) $\min / 1.73 \mathrm{~m}^{2}$ ) was more stringent compared with that of the ACR guideline and overseas hospitals, which used using $30 \mathrm{~mL} / \mathrm{min} / 1.73 \mathrm{~m}^{2}(5,10)$, and with that of the Korean guideline, which recommends $45 \mathrm{~mL} / \mathrm{min} / 1.73 \mathrm{~m}^{2}$.

Because metformin-associated lactic acidosis after ICM exposure is significantly rarer than previously believed, relaxed guidelines have been proposed in recent years (11, 12). The ACR and ESUR guidelines have lower cut-offs (eGFR, $30 \mathrm{~mL} / \mathrm{min} / 1.73 \mathrm{~m}^{2}$ and $45 \mathrm{~mL} / \mathrm{min} / 1.73 \mathrm{~m}^{2}$, respectively). However, $57.1 \%$ of the Korean hospitals asked patients to discontinue metformin, regardless of renal function and, even in these institutions, the policies were significantly more stringent and failed to reflect up-to-date guidelines.

Before ICM exposure, a fasting policy was significantly more stringent in Korean hospitals compared with overseas hospitals. Because current guidelines recommend the intake of fluid before ICM exposure, and for solid-food fasting, it is recommended that the requirements should be adjusted according to patient circumstances. This is because the benefit of fasting was questioned before the administration of non-ionic ICM, which induced fewer adverse reactions than ionic $\operatorname{ICM}(13,14)$.

Although most Korean hospitals had basic emergency equipment in the CT room, fewer Korean hospitals were equipped with a bronchodilator than overseas hospitals $(p=0.004)$. Furthermore, $<90 \%$ of Korean hospitals were equipped with epinephrine, an intubation kit, and a defibrillator, which are essential for managing anaphylaxis to ICM. The administration of ICM during night-shift hours was more frequently performed without the observation of medical staff in Korean hospitals than in overseas hospitals $(p=0.026)$. Given the results, there is room for improving management of acute adverse reactions to ICM in Korean hospitals.

For the management of these adverse reactions to ICM, Korean hospital protocols more frequently included content regarding the future use of ICM in patients with a previous adverse reaction to ICM than in other countries $(p=0.001)$, but tended to less frequently address immediate drug administration $(86.4 \%$ versus $100 \% ; p=$ $0.195)$ or responding to a delayed reaction $(62.7 \%$ versus $73.3 \% ; p=0.442$ ). This is due to the fact that delayed reactions are, in large part, self-limiting, and their clinical significance tends to be overlooked compared with acute 
adverse reactions (15). Given that the incidence of delayed reactions may be higher than is generally recognized (16), instructions for managing delayed reactions require further supplementation.

Among the Korean hospitals with an institutional protocol for patients with a history of adverse reactions to ICM, the causative ICM was replaced with another ICM in $58.3 \%$ and, in fact, the strategy was useful for reducing the incidence of adverse reactions on repeated exposure to ICM (1719). On the other hand, a skin test was rarely included in the protocol $(17 \%)$. This may be due to a paucity of experienced allergists and the lack of a referral system to perform skin tests in those who would benefit from them. Recent studies have suggested that skin tests can help to identify the causative ICM, especially for patients with severe reactions to ICM (20).

Our study had some limitations. First, although many hospitals participated in the study, one-quarter of Korean referral hospitals were not included. In addition, 15 overseas hospitals were arbitrarily chosen by the authors' professional connections for comparison. Thus, the limited number and biased selection of overseas hospitals surveyed may be insufficiently representative of international status. For further investigation, a nationwide survey including not only referral hospitals, but also regional clinics, would enable capturing the status of managing adverse reactions to ICM at the country level in the future. Second, this study focused on the management of adverse reactions to ICM; however, the reaction can occur to gadolinium contrast media for magnetic resonance imaging (21). Gadolinium contrast media are also known to provoke adverse reactions; therefore, further investigation is necessary $(22,23)$. Finally, our results were fully based on answers to questionnaires rather than detailed analysis of the individual guideline document in the included institutions, because the acquisition of documents is not practically possible due to institutional security and privacy policies.

In conclusion, for the management of ICM, Korean hospitals have established systematic protocols and have adhered relatively well to identifying risk factors, responding to acute adverse reactions, and managing patients with a history of adverse reactions to ICM. However, an institutional protocol for evaluating renal function varied across the hospitals, with limited adherence to up-to-date guidelines, and there was a lack of protocols for managing delayed reactions. In addition, the cut-off value for renal function, metformin-taking policy, and pre- test fasting policy were strict and did not adequately reflect recent research compared with current guidelines and overseas hospitals. Finally, it may be necessary to actively use skin testing and replacement of ICM in patients with a history of an adverse reaction to ICM, reflecting recent findings.

\section{Supplementary Materials}

The online-only Data Supplement is available with this article at https://doi.org/10.3348/kjr.2017.0771.

\section{Conflicts of Interest}

The authors have no financial conflicts of interest.

ORCID

Soon Ho Yoon

https://orcid.org/0000-0002-3700-0165

Seungchul Han

https://orcid.org/0000-0002-7276-0370

\section{REFERENCES}

1. Bottinor W, Polkampally P, Jovin I. Adverse reactions to iodinated contrast media. Int J Angiol 2013;22:149-154

2. Bae K, Lee SM, Ha JY, Jeon KN, Moon JI, Choi BH, et al. Adverse drug reactions to CT contrast media in South Korea: incidence and risk factors. J Korean Soc Radiol 2016;75:41-48

3. Bata P, Tarnoki AD, Tarnoki DL, Horvath E, Berczi V, Szalay F. Acute severe thrombocytopenia following non-ionic lowosmolarity intravenous contrast medium injection. Korean J Radiol 2012;13:505-509

4. CT and X-ray contrast guidelines. UCSF Department of Radiology \& Biomedical imaging Web site. https://radiology. ucsf.edu/patient-care/patient-safety/contrast/iodinated. Updated July 20, 2017. Accessed September 17, 2017

5. ACR Committee on Drugs and ContrastMedia. ACR manual on contrast media version 10.3. 2017. Available at: https://www. acr.org/-/media/ACR/Files/Clinical-Resources/Contrast_Media. pdf. Accessed September 17, 2017

6. Korean Society of Radiology, Korean Academy of Asthma, KIoDSRM. Korean clinical practice guideline on adverse reactions of injectable iodinated contrast agent and gadolinium contrast agent for MRI, 2nd edition. [Published 2016]. Available at: http://www.allergy.or.kr/file/ allergic2016.pdf. Accessed September 17, 2017

7. Drug safety information report trend. Korean Institute of Drug Safety \& Risk Management imaging Web site. http://open. drugsafe.or.kr/trend/trend/Read.jsp?ntt_id=2003. Published 2016. Accessed September 17, 2017 
8. ESUR guidelines on contrast media. Version 8.1. European Society of Urogenital Radiology Web site. http://www.esur. org/guidelines/. Published 2013. Accessed September 17, 2017

9. Lee J, Cho JY, Lee HJ, Jeong YY, Kim CK, Park BK, et al.; Korean Society of Urogenital Radiology (KSUR); Korean Society of Radiology. Contrast-induced nephropathy in patients undergoing intravenous contrast-enhanced computed tomography in Korea: a multi-institutional study in 101487 patients. Korean J Radiol 2014;15:456-463

10. Davenport MS, Khalatbari S, Cohan RH, Dillman JR, Myles JD, Ellis JH. Contrast material-induced nephrotoxicity and intravenous low-osmolality iodinated contrast material: risk stratification by using estimated glomerular filtration rate. Radiology 2013;268:719-728

11. Salpeter SR, Greyber E, Pasternak GA, Salpeter EE. Risk of fatal and nonfatal lactic acidosis with metformin use in type 2 diabetes mellitus. Cochrane Database Syst Rev 2010;(4):CD002967

12. Stang M, Wysowski DK, Butler-Jones D. Incidence of lactic acidosis in metformin users. Diabetes Care 1999;22:925-927

13. Oowaki K, Saigusa H, Ojiri H, Ariizumi M, Yamagisi J, Fukuda $K$, et al. [Relationship between oral food intake and nausea caused by intravenous injection of iodinated contrast material]. Nihon Igaku Hoshasen Gakkai Zasshi 1994;54:476479

14. Wagner HJ, Evers JP, Hoppe M, Klose KJ. [Must the patient fast before intravascular injection of a non-ionic contrast medium? Results of a controlled study]. Rofo 1997;166:370375

15. Webb JA, Stacul F, Thomsen HS, Morcos SK; Members Of The Contrast Media Safety Committee Of The European Society of
Urogenital Radiology. Late adverse reactions to intravascular iodinated contrast media. Eur Radiol 2003;13:181-184

16. Loh S, Bagheri S, Katzberg RW, Fung MA, Li CS. Delayed adverse reaction to contrast-enhanced CT: a prospective single-center study comparison to control group without enhancement. Radiology 2010;255:764-771

17. Abe S, Fukuda H, Tobe K, Ibukuro K. Protective effect against repeat adverse reactions to iodinated contrast medium: premedication vs. changing the contrast medium. Eur Radiol 2016;26:2148-2154

18. Park HJ, Park JW, Yang MS, Kim MY, Kim SH, Jang GC, et al. Re-exposure to low osmolar iodinated contrast media in patients with prior moderate-to-severe hypersensitivity reactions: a multicentre retrospective cohort study. Eur Radiol 2017;27:2886-2893

19. Lee SY, Yang MS, Choi YH, Park CM, Park HW, Cho SH, et al. Stratified premedication strategy for the prevention of contrast media hypersensitivity in high-risk patients. Ann Allergy Asthma Immunol 2017;118:339-344.e1

20. Yoon SH, Lee SY, Kang HR, Kim JY, Hahn S, Park CM, et al. Skin tests in patients with hypersensitivity reaction to iodinated contrast media: a meta-analysis. Allergy 2015;70:625-637

21. Behzadi AH, Zhao Y, Farooq Z, Prince MR. Immediate allergic reactions to gadolinium-based contrast agents: a systematic review and meta-analysis. Radiology 2018;286:471-482

22. Kanal E, Barkovich AJ, Bell C, Borgstede JP, Bradley WG Jr, Froelich JW, er al.; ACR Blue Ribbon Panel on MR Safety. ACR guidance document for safe MR practices: 2007. AJR Am J Roentgenol 2007;188:1447-1474

23. Perazella MA. Current status of gadolinium toxicity in patients with kidney disease. Clin J Am Soc Nephrol 2009;4:461-469 